\title{
The long helical jet of the Lighthouse nebula, IGR J11014-6103
}

\author{
L. Pavan ${ }^{1}$, P. Bordas ${ }^{1,2}$, G. Pühlhofer ${ }^{2}$, M. D. Filipović ${ }^{3}$, A. De Horta ${ }^{3}$, A. O’ Brien ${ }^{3}$, M. Balbo ${ }^{1}$, R. Walter ${ }^{1}$, \\ E. Bozzo ${ }^{1}$, C. Ferrigno ${ }^{1}$, E. Crawford ${ }^{3}$, and L. Stella ${ }^{4}$
}
1 ISDC Data Center for Astrophysics, Université de Genève, 16 chemin d'Ecogia, 1290 Versoix, Switzerland e-mail: Lucia.Pavan@unige.ch
2 Institut für Astronomie und Astrophysik, Universität Tübingen, Sand 1, 72076 Tübingen, Germany
3 Computational Astrophysics, Imaging \& Simulation School of Computing \& Mathematics, University of Western Sydney, 2006 Sydney, Australia
${ }^{4}$ INAF - Osservatorio astronomico di Roma, via di Frascati 33, 00040 Monte Porzio Catone, Roma, Italy

Received 2 September 2013 / Accepted 12 January 2014

\begin{abstract}
Context. Jets from rotation-powered pulsars so far have only been observed in systems moving subsonically through their ambient medium and/or embedded in their progenitor supernova remnant (SNR). Supersonic runaway pulsars are also expected to produce jets, but they have not been confirmed to so far.

Aims. We investigated the nature of the jet-like structure associated with the INTEGRAL source IGR J11014-6103 (the "Lighthouse nebula"). The source is a neutron star escaping its parent SNR MSH 11-61A supersonically at a velocity exceeding $1000 \mathrm{~km} \mathrm{~s}^{-1}$.

Methods. We observed the Lighthouse nebula and its jet-like X-ray structure through dedicated high spatial resolution observations in X-rays (with Chandra) and in the radio band (with ATCA).

Results. Our results show that the feature is a true pulsar's jet. It extends highly collimated over $\gtrsim 11 \mathrm{pc}$, displays a clear precessionlike modulation, and propagates nearly perpendicular to the system direction of motion, implying that the neutron star's spin axis in IGR J11014-6103 is almost perpendicular to the direction of the kick received during the supernova explosion.

Conclusions. Our findings suggest that jets are common to rotation-powered pulsars, and demonstrate that supernovae can impart high kick velocities to misaligned spinning neutron stars, possibly through distinct, exotic, core-collapse mechanisms.
\end{abstract}

Key words. X-rays: individuals: IGR J11014-6103 - stars: jets - stars: neutron - supernovae: individual: MSH 11-61A ISM: jets and outflows - ISM: supernova remnants

\section{Introduction}

Pulsar wind nebulae (PWNe) powered by pulsars that are still embedded in their progenitor supernova remnant (SNR) are typically seen as extended cocoons, in some cases accompanied by two collimated jets (Weisskopf et al. 2000; Gaensler \& Slane 2006; Kargaltsev \& Pavlov 2008; Durant et al. 2013). As of yet, jets have not been clearly identified from runaway pulsars that are traveling supersonically through the interstellar medium, which show otherwise cometary-like features formed by the confinement of their winds by a bow-shock (Gaensler \& Slane 2006). Deep statistical studies have proved that pulsar's spin axes (and hence jets, when present) are generally aligned with their proper motion (Weisskopf et al. 2000; Johnston et al. 2007; Durant et al. 2013), a relation that has been explained by numerous theoretical models of asymmetric supernova mechanisms (Spruit \& Phinney 1998; Lai et al. 2001; Janka 2012). Jets in runaway systems could therefore be either disrupted by the system motion or aligned with (and hidden by) the bow-shocked PWN (Kargaltsev \& Pavlov 2008), making their detection difficult.

In a few cases only, puzzling jet-like X-ray features extending over parsec-scales have been observed in association with high velocity pulsars, e.g., in the Guitar nebula (powered by pulsar PSR B2224+65; Hui et al. 2012) and in Morla (powered by PSR J0357+3205; De Luca et al. 2013). In the latter case, the jet-like feature is aligned with the pulsar proper motion, and may correspond to a trail of thermal emission from the shocked ambient medium (Marelli et al. 2013). In the Guitar nebula, in contrast, the collimated X-ray outflow is inclined by $\sim 118^{\circ}$ with respect to the backwards $\mathrm{PWN}$, which is seen in the optical band as a bright $\mathrm{H}-\alpha$ nebula (Cordes et al. 1993). Although observations performed at different epochs confirmed the link between this extended X-ray feature and the pulsar (Johnson \& Wang 2010), its nature is still under discussion, and different scenarios, including a truly ballistic jet (Johnson \& Wang 2010; Hui et al. 2012) or a flow of high-energy electrons diffusing into the ambient magnetic field (Bandiera 2008), have been proposed.

The INTEGRAL source IGR J11014-6103, which we call the Lighthouse nebula, located $11^{\prime}$ southwest of the SNR MSH 11-61A (estimated distance 7 1 kpc, Reynoso et al. 2006), is a complex system that, in X-rays, displays a point source, an elongated cometary tail, and perpendicular to this, a prominent jet-like feature (see Pavan et al. 2011, hereafter Paper I; and Tomsick et al. 2012, T12 in the following). While the previous studies already suggested a PWN scenario for the cometary tail in IGR J11014-6103, the jet-like feature remained as yet uninterpreted due to the poor statistics of the available data. To unveil its real nature, we obtained high spatial resolution observations of the Lighthouse nebula with the Chandra X-ray Observatory and in the radio band with the Australia Telescope Compact Array (ATCA). 


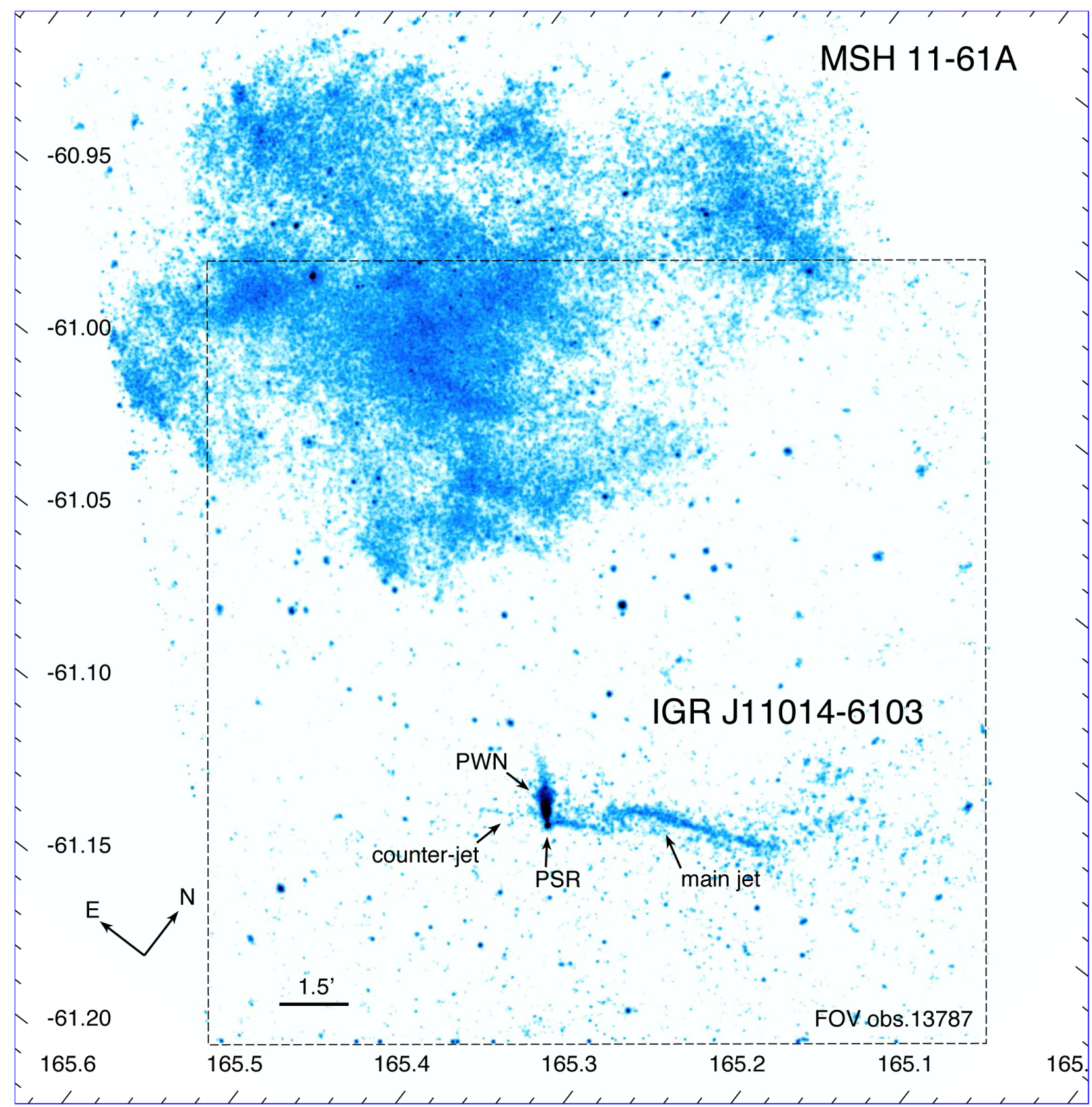

Fig. 1. Lighthouse nebula (IGR J11014-6103) as viewed by Chandra during our observation (obs.ID 13787, with the field of view indicated by the dashed box). The image also includes archival observations of SNR MSH 11-61A. The two objects have likely been produced by the same supernova explosion 10-20 kyr ago. The image is adaptively smoothed with a gaussian kernel, rotated by $37.5^{\circ}$, and normalized by the exposure to recover gaps between the chips. Labels indicate the terms used throughout the text for the different components of the Lighthouse nebula.

\section{Observations and data analysis}

\subsection{Chandra X-ray observation}

We observed the Lighthouse nebula with Chandra for $50 \mathrm{ks}$ on October 11, 2012 (obs.ID 13787). The data have been analyzed with the Chandra Interactive Analysis of Observations package (CIAO version 4.5; Fruscione et al. 2006) and reprocessed with standard tools using the most recently available calibration, as recommended by the Chandra team. We applied standard filters to the event file, on grade, status, and good time intervals. The observation was not affected by strong background flares, resulting in a final net exposure of $49.4 \mathrm{ks}$.

We performed the observation with the Advanced CCD Imaging Spectrometer (ACIS-I) detector pointing at source
2XMM J110145.0-610140 ("PSR” hereafter, see Fig. 1), with a moderate offset of $0.8^{\prime}$ with respect to the nominal aim point, to optimize the results for the imaging of the PSR itself, the cometary PWN, and jet structures. All components of the Lighthouse nebula and a large portion of the nearby SNR MSH 11-61A are included in the total ACIS field of view (FOV, see dashed box in Fig. 1).

\subsubsection{Imaging}

Chandra observations in imaging mode are performed with a dithering technique that enables coverage of the gaps between CCDs, though these are characterized by a reduced net exposure. To recover these sections, we computed a fluxed image, by 
Table 1. Best fit spectral parameters for the Lighthouse nebula components.

\begin{tabular}{lcccc}
\hline \hline & $\begin{array}{c}N_{\mathrm{H}} \\
\left(10^{22} \mathrm{~cm}^{-2}\right)\end{array}$ & $\Gamma$ & $\begin{array}{c}F_{2-10 \mathrm{keV}} \\
\left(10^{-13} \mathrm{erg} \mathrm{cm}^{-2} \mathrm{~s}^{-1}\right)\end{array}$ & $\tilde{\chi}^{2} /$ d.o.f. \\
\hline PSR & $1.0 \pm 0.2$ & $1.1 \pm 0.2$ & $6.1 \pm 0.6$ & $1.09 / 70$ \\
PWN & $0.8 \pm 0.1$ & $1.9 \pm 0.1$ & $6.7 \pm 0.5$ & $0.82 / 76$ \\
Main jet & $0.8 \pm 0.2$ & $1.6 \pm 0.2$ & $5.4 \pm 0.5$ & $1.08 / 65$ \\
\hline
\end{tabular}

Notes. All spectra were fit using an absorbed power-law model (photon index $\Gamma$ ). Uncertainties are at $90 \%$ c.l. on the spectral parameters and $68 \%$ c.l. on the fluxes.

normalizing the count map with the local net exposure. The exposure map takes the Chandra effective area into account, and is therefore energy-dependent. We thus computed the instrument and exposure maps, weighting them for the spectral distribution observed from the PSR source (see Sect. 2.1.3 and Table 1), through the standard CIAO tools make_instmap_weights and mkinstmap. We verified that different, reasonable weights for the instrument map did not significantly affect the resulting corrected image (Fig. 1). We detected X-ray extended emission up to $3 \mathrm{keV}$ from the SNR MSH 11-61A in agreement with what has been previously reported by García et al. (2012). The three components of the Lighthouse nebula were clearly visible in our Chandra observation. We detected PSR at RA 11:01:44.915, Dec -61:01:38.66 (J2000, associated uncertainty $\left.0.64^{\prime \prime}\right)$ in agreement with the position previously reported by T12. The PWN is detected up to $1.2^{\prime}$ from PSR. The main jet is clearly detected up to $\sim 5.5^{\prime}$ from PSR in a northwest direction, forming an angle of $\sim 104^{\circ}$ with the PWN axis. The Chandra data also revealed for the first time that the long jet is highly collimated and displays a strikingly well-defined corkscrew modulation. In the corrected image, the jet shows a remarkable change of orientation at about $1.4^{\prime}$ from PSR. We did not attempt a more refined analysis and interpretation of this region as it lies in a gap between two CCDs, and thus is characterized by a much lower net exposure time with respect to the surroundings.

In addition to these three components, our observation has revealed a fainter linear region opposite the jet, in a southeast direction (see Fig. 1). This extended region is clearly detected with the Voronoi Tessellation and Percolation source detection (CIA0/vtpdetect) algorithm up to $1.5^{\prime}$ from PSR, with a detection significance of $3.7 \sigma$. The orientation and position of this linear feature is readily interpreted as a counterjet.

The counterjet is well aligned with the direction of the main jet within the first arcminute from PSR. Both features intersect the PWN at 7.4" from the point source. Within $30^{\prime \prime}$ from the PSR, however, the jet bends directly toward the point source and is smoothly connected to it (see Fig. 1). The alignment between the jet and counterjet, together with the change of brightness at the PSR location and their smooth connection to PSR, rule out any chance coincidence between these structures.

\subsubsection{Point-like source PSR}

To verify the point-like nature of PSR, we extracted the radial intensity profile of the source (background subtracted) and compared it to the corresponding instrumental point-spread function (PSF). The PSF was obtained by simulations performed with ChaRT (Carter et al. 2003), assuming the spectral profile of the PSR source (see Table 1) and reprojected with MARX v.5
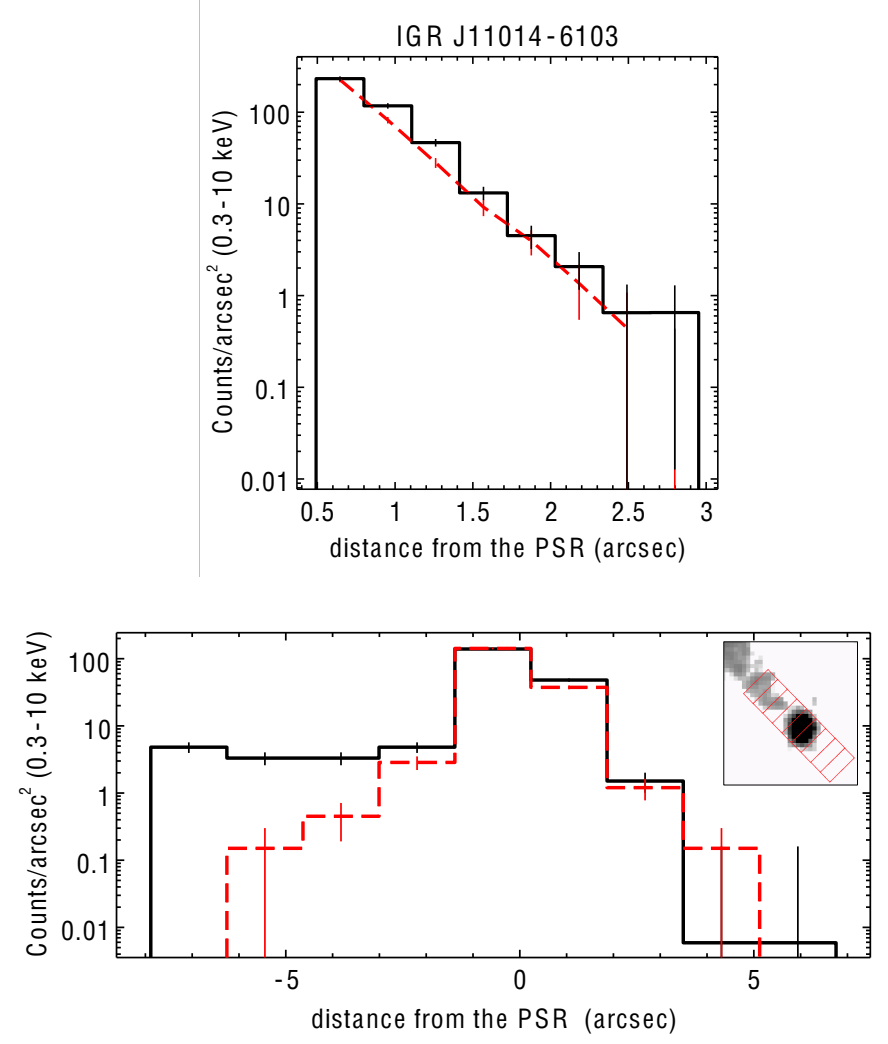

Fig. 2. Top panel: radial profile within $3^{\prime \prime}$ from the source PSR (histogram in black) compared to the PSF (dashed red line) simulated with the ChaRT tool at the PSR position and with the measured energy spectrum. Bottom panel: same plot as above, but for the regions marked in the inset picture. Positive radial distances are in front of the source, in the SW direction, negative are backwards in the NE direction (i.e. in the direction of the PWN).

(Davis et al. 2012) to the observed source position on the ACISI3 chip. The observed and simulated profiles, extracted within a region of $3^{\prime \prime}$ around PSR, are displayed in the upper panel of Fig. 2. We verified that there are no variations on the profile between the front and rear regions. Similarly, we extracted the intensity profile along the axis of the PWN from a rectangular region centered on the source and extending up to $7^{\prime \prime}$ (Fig. 2, lower panel). The comparison with the simulated PSF shows that there is no detectable extension in front of the source, and that the PWN is smoothly connected to the point source, emerging $3^{\prime \prime}$ away from it already.

The relatively poor timing resolution of the Chandra data in timed exposure mode $(3.2 \mathrm{~s})$ did not permit us to analyze the timing periodicity of the point source. Previous searches with XMM-Newton (Paper I) and Parkes (T12) did not reveal any significant coherent periodicity. We note, however, that in almost one-third of the known PWN systems there is no clear pulsating signal from the neutron star (see, e.g., Roberts 2004). This can be due to geometrical phenomena, when the beam of light from the pulsar is oriented in a direction not intercepting Earth, as well as physical phenomena, if the presence of a dense region surrounding the pulsar prevent the direct observation of the neutron star surface and its pulsations (Kargaltsev \& Pavlov 2008).

\subsubsection{Spectra}

We extracted background-corrected spectra of the different components of the Lighthouse nebula with the standard 
CIAO/specextract tool, and analyzed them with xspec (v.12.7.1; Arnaud 1996).

All the extended components and PSR were comprised in the FOV of ACIS I3. The main jet, which was comprised in ACIS I2, was the only exception. Therefore, the background regions were chosen from source free areas in ACIS chips I2 and I3. We verified that different reasonable choices of the background did not significantly affect our spectral results.

We extracted the spectrum of PSR from a circular region of 2.3" centered on the source to avoid contamination from the PWN and the jets. We created the corresponding response matrix file (RMF) and energy-corrected ancillary response file (ARF) with the CIAO standard tools. The spectrum was grouped to have at least 15 counts per energy bin. For the jet and PWN, we followed standard CIAO spectral extraction procedure for extended sources. We used specextract to compute weighted RMFs and ARFs for each component.

All spectra could be fit with a simple absorbed power-law model, with best-fit parameters reported in Table 1. We did not attempt any spectral extraction for the counterjet, given the low number of counts. In addition to the previous jet analysis, we extracted spectra from different rectangular regions along the jet, to search for possible spectral variations. The data, however, did not reveal any spectral variation between the various regions, within the uncertainties. The source PSR showed a power-law photon index of $\Gamma=1.1 \pm 0.2$, which is compatible with that expected from young energetic pulsars surrounded by their wind nebulae (Gotthelf \& Halpern 2008). A softer spectrum is obtained for the main jet and the PWN, with $\Gamma=1.6 \pm 0.2$ and $1.9 \pm 0.1$, respectively. For the PWN, such a relatively soft spectrum is expected (see, e.g., Gotthelf 2004; and Fig. 8 in Li et al. 2008).

We also tentatively fitted a pure thermal emission model (vmekal model in xspec) to the jet spectrum (see, e.g., the adiabatic expansion scenario for the precessing jets of SS 433, Migliari et al. 2002). This model provides a good fit to the data, $\left(\tilde{\chi}^{2}=1.09 / 65\right.$ d.o.f., similar to that obtained with the power-law model), with best fit parameters $N_{\mathrm{H}}=(0.8 \pm 0.2) \times 10^{22} \mathrm{~cm}^{-2}$ and $k T=11_{-5}^{+17} \mathrm{keV}$. Although line emission should be expected at such temperatures, in particular around 6-7 keV, the relatively low count statistics of the jet spectrum prevents us from probing the existence of such lines. Our fit results, therefore, cannot clearly distinguish between a pure thermal and a power-law model (a fit using both components provides a slightly worse $\tilde{\chi}^{2}$ and is unable to constrain neither the plasma temperature nor the value of $N_{\mathrm{H}}$ ). We note, however, that in a thermal scenario the plasma is expected to cool down on length-scales much shorter than the $\sim 11 \mathrm{pc}$ displayed by the main jet, and, therefore, a continuous reheating of the emitting material would be required to explain the observed lack of spectral changes along the jet. In addition, pulsar jets are supposed to have a magnetohydrodynamical origin (see, e.g., Bogovalov \& Tsinganos 1999), and therefore a power-law component from synchrotron emission should in any case be expected (see, e.g., Pavlov et al. 2003; Johnson \& Wang 2010; De Luca et al. 2011; Hui et al. 2012). We conclude that the thermal model is disfavored with respect to the power-law model, and a synchrotron scenario is assumed in the following.

The jet feature and the counterjet together provide almost $1 / 3$ of the total X-ray flux $\left(\sim 2 \times 10^{-12} \mathrm{erg} \mathrm{cm}^{-2} \mathrm{~s}^{-1}\right)$ of the Lighthouse nebula.

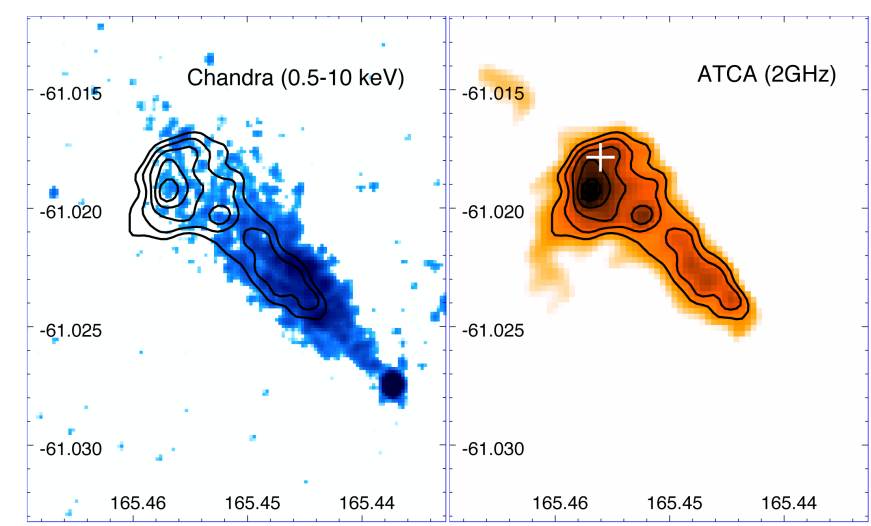

Fig. 3. Chandra (left) and ATCA $2 \mathrm{GHz}(r i g h t)$ images of the PWN. The ATCA contours are overplotted on both images. On the right panel, a cross marks the position of the radio source as reported in the MGPS-2 survey (see text for details).

\subsection{ATCA radio observations}

We observed the Lighthouse nebula region on January 11, 2013 and February 22, 2013 with ATCA (project C2651), using the new Compact Array Broadband Backend (CABB) receiver at array configurations of EW352 and 6A, and wavelengths of 20, 6, and $3 \mathrm{~cm}(v=2,5.5$ and $9 \mathrm{GHz})$, with bandwidths of $2 \mathrm{GHz}$. The observations were carried out in "change-frequency" mode, totaling $\sim 24 \mathrm{~h}$ of integration over the two observing periods. The sources PKS B1934-638 and PKS 1059-63 were used for primary and secondary (phase) calibration, respectively.

We used the miriad (Sault et al. 1995) and karma (Gooch 1996) software packages for reduction and analysis. Images were formed using miriad multifrequency synthesis (Sault et al. 1995) and natural weighting. They were deconvolved using the mfclean and restor algorithms with primary beam correction applied using the linmos task. We used a similar procedure for both $U$ and $Q$ Stokes parameter maps. Because of the good dynamic range (signal-to-noise ratio between the source flux and $3 \sigma$ noise level), we applied self-calibration, which resulted in our best total intensity image (see Fig. 3). While our shortest baseline was $46 \mathrm{~m}$ (at EW352 array), we still suffered from the missing flux from the lack of short (zero) spacings. This effect was far more pronounced at the higher frequencies: at $v=5.5$ and $9 \mathrm{GHz}$, the observations were significantly affected by this "missing short spacing" and, therefore, were excluded from the analysis, which was then performed only in the $2 \mathrm{GHz}$ band.

The candidate radio counterpart MGPS J110149-610104 (Paper I) was clearly detected in our $2 \mathrm{GHz}$ image as an extended source (the image resolution is $5.177^{\prime \prime} \times$ $4.146^{\prime \prime}$ at $\mathrm{PA}=-8.5828^{\circ}$ with an estimated rms noise of $0.15 \mathrm{mJy}^{-1}$ beam $^{-1}$ ). Its extension and position coincide well with the X-ray contours of the PWN (Fig. 3), the source extends up to $80^{\prime \prime}$ from PSR (Fig. 4). Its total integrated flux density is $23 \pm 2 \mathrm{mJy}$ at $2 \mathrm{GHz}$. There was no reliable detection in the $Q$ and $U$ intensity parameters at any observed frequency that could be associated with this object. Without these detections, the Faraday rotation and consequently the magnetic field properties could not be determined.

The PSR and the jets were not detected in the $2 \mathrm{GHz}$ image, providing an upper limit of $0.45 \mathrm{mJy} \mathrm{beam}^{-1}$ at a $3 \sigma$ confidence level. For the jet, this translates into a total flux, integrated over the region used for the $\mathrm{X}$-ray spectral extraction (a boxed region of size $228.6^{\prime} \times 40.5^{\prime}$ ), lower than $\sim 4 \times 10^{-15} \mathrm{erg} \mathrm{cm}^{-2} \mathrm{~s}^{-1}$. 


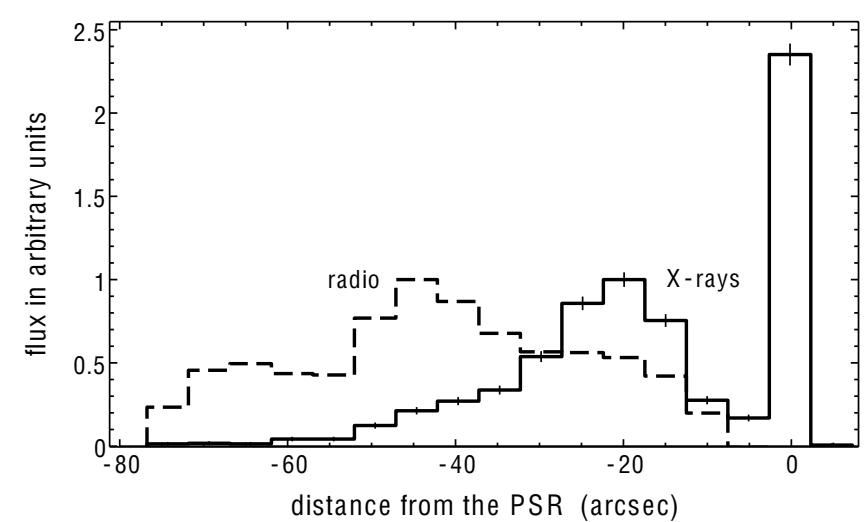

Fig. 4. Intensity profiles along the $\mathrm{PWN}$, at $2 \mathrm{GHz}$ (dashed line) and $0.5-10 \mathrm{keV}$ (solid line), as a function of the distance from PSR. The radio and X-ray profiles peak at different positions, separated by $22^{\prime \prime}$. This can be explained by the cooling of the emitting particles. The shape of the PWN at both wavebands and its alignment are in favor of a high pulsar velocity $\left(>1000 \mathrm{~km} \mathrm{~s}^{-1}\right)$.

\subsection{Simplified precession model}

Our Chandra observation shows the presence of a jet and a counterjet, both well collimated, with different brightness and elongation. This might be interpreted as due to the different orientation with respect to the line of sight under the assumption that the jet and counterjet are intrinsically identical in terms of luminosity and extension. The data also revealed a clear corkscrew modulation of the main jet, reminiscent of that seen in other galactic jets (see, e.g., Stirling et al. 2002; Durant et al. 2013), which can be interpreted either as precession of the neutron star or as kink instabilities in a ballistic jet (see in Sect. 3).

To further investigate this scenario, we used a simplified precession model, which describes the large-scale structure of the jet. We considered particles moving linearly away from the central position, each of them launched toward a direction lying on the precession cone surface (see Fig. 5). In the absence of any external effect, the interpolation of all particles forms a conical helix. We let the semi-aperture angle of the cone, the period of precession, the phase angle of the helix, and the velocity of the particles moving along the helix, as free parameters. In this model, all particles move at the same velocity, equal to the bulk velocity of the jet. The section of the helix has a $2 \mathrm{D}$ gaussian profile to simulate the degree of collimation of the jet. The width of this gaussian is another free parameter.

All of the model parameters are strongly dependent on the particles' velocity $\beta c$ (where $c$ is the speed of light), therefore, we kept this parameter frozen and performed simulations for different values of $\beta$. The velocity vector of each particle is used to determine the Doppler boosting factor of the emitted radiation $\delta=\gamma^{-1}\left(1-\beta \cos \theta_{\mathrm{i}}\right)^{-1}$ (where $\gamma=\left(1-\beta^{2}\right)^{-1 / 2}$ is the Lorentz factor of the particle and $\theta_{\mathrm{i}}$ is the angle to the observer).

We fixed the position of the jet base in image coordinates to match the observed launching point of the jet. The 3D direction of the precession axis is constrained by the ratio $L_{\text {counterjet }} / L_{\text {jet }} \sim$ 0.05 observed between jet and counterjet apparent luminosities. In this scenario, the $L_{\text {counterjet }} / L_{\text {jet }}$ ratio is due to the different orientation of the two jets, and to the relativistic beaming effects due to the bulk motion of the emitting particles. Following the same Doppler boosting prescription as above, at each bulk velocity $\beta$ we find the corresponding angle between the jet axis and the line of sight $\theta_{\mathrm{i}}$ needed to reproduce the observed

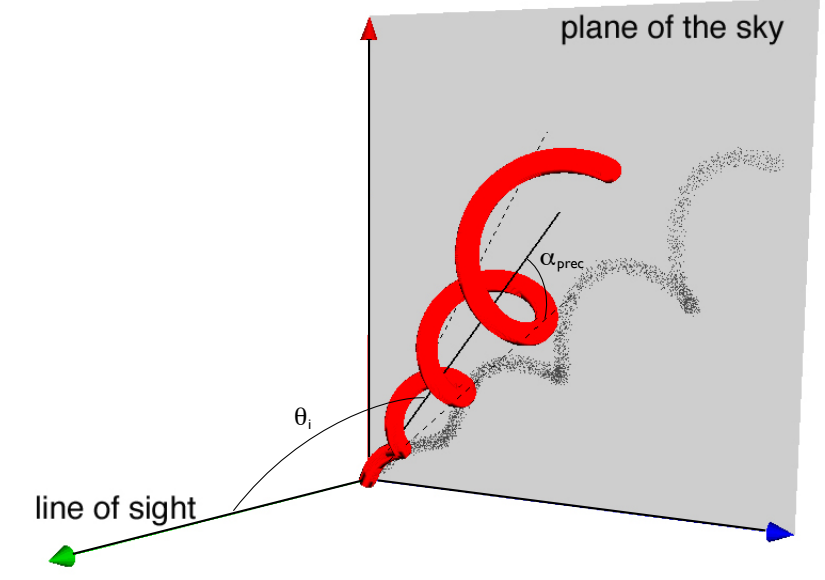

Fig. 5. Sketch of the precession model. The semi-aperture angle of the precession cone is marked as $\alpha_{\text {prec }}$. The angle between the jet axis and the line of sight $\theta_{\mathrm{i}}$ is also shown. The projection of the $3 \mathrm{D}$ helix on the plane of the sky (shown here in grey) is used to fit the Chandra image after being corrected for the Doppler factors of each particle.

ratio $L_{\text {counterjet }} / L_{\text {jet }}$. We then derive a lower limit for the velocity of the particles along the jet axis $v_{\text {jet, } \|} \gtrsim 0.52 c\left(\sin \theta_{\mathrm{i}}\right)^{-1}$. The projection of the helix on the plane of the sky, corrected for the Doppler boosting factor of each particle, is then fit to the data with the Sherpa package (Freeman et al. 2001), using the Nelder-Mead Simplex optimization method with Cash statistics (see Fig. 6). We explored several values of the bulk velocity in the permitted range $\beta=v_{\text {jet }, \|} / c \geq 0.52$.

After the 2D spatial fit is performed, we compare the brightness modulation observed in our Chandra data along the jet (see inset in Fig. 6) to the modulation obtained from the model, which is given by approaching/receding relativistic beaming effects, and therefore, provides three-dimensional information of the jet morphology.

The minimum bulk velocity $\beta=0.52$, which corresponds to a face-on jet with $\theta_{\mathrm{i}}=0$, is excluded by the observed elongation of the jet and the presence of the counterjet in the Chandra data. The fit also provides a more stringent limit $\beta \gtrsim 0.7$, since an inclination angle $\theta_{\mathrm{i}} \gtrsim 40^{\circ}$ is required to match the jet morphology and the Doppler boosting effects on the brightness profile. Within this refined range, a value $\beta \sim 0.8$ is favored (see Fig. 6), resulting in a jet inclined by $\theta_{\mathrm{i}} \sim 50^{\circ}$ to the line of sight (with an intrinsic length of the main jet of $\sim 15 \mathrm{pc}$ ), a semi-aperture angle of the precessing cone of $4.5^{\circ}$ and a precession period of nearly $66 \mathrm{yr}$. The relatively low signal to noise ratio of the image did not permit us to estimate the corresponding uncertainties.

\section{Discussion}

Besides the pulsar, the PWN, and the perpendicular jet-like feature described in earlier works, our Chandra observation has revealed the presence of a counterjet extending in the opposite direction from that of the main jet for about $1.5^{\prime}$. The Chandra data also showed for the first time that the long jet is highly collimated and displays a strikingly well-defined and continuous corkscrew modulation, with a length of $\sim 5.5^{\prime}$. The column density estimated in X-rays for the different components of the Lighthouse nebula $\left(N_{\mathrm{H}}=[0.8 \pm 0.2] \times 10^{22} \mathrm{~cm}^{-2}\right.$, see Table 1) is in good agreement with that found in various regions of the neighboring SNR MSH 11-61A $\left([0.4-0.7] \times 10^{22} \mathrm{~cm}^{-2}\right.$; 


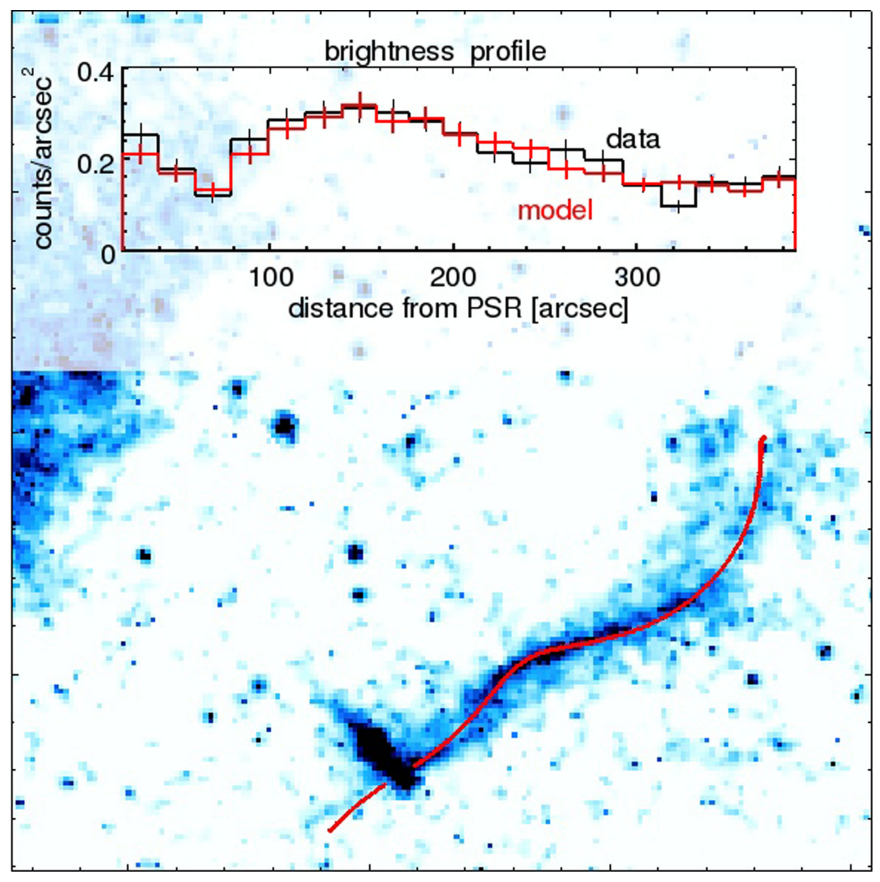

Fig. 6. Best-fit model of a precessing jet (solid red line) overplotted on top of the Chandra image of the Lighthouse nebula. The plot (and the inset) is relative to particles with bulk velocity $\beta=0.8$ (see text for details). The inset shows the brightness profile measured along the jet (in black), and the brightness profile obtained from the model (in red in the online version).

García et al. 2012), proving the distance of the Lighthouse nebula to be $\sim 7 \mathrm{kpc}\left(d_{7}\right)$.

The radio source MGPS J110149-610104, previously identified as a possible counterpart of the Lighthouse nebula (Paper I), is clearly detected in our ATCA observation. Its morphology closely matches the X-ray PWN (see Fig. 3). The flux density measured at $2 \mathrm{GHz}(23 \pm 2 \mathrm{mJy})$ is compatible with the flux density reported at $843 \mathrm{MHz}$ in the MGPS-2 archive (24 $\pm 5 \mathrm{mJy}$; see Paper I). This indicates that the tail has a flat radio spectrum, i.e., with radio spectral index $\alpha \sim 0$, as typically observed in PWNe (Gaensler \& Slane 2006). The neutron star and jet structures are not detected in radio (upper limit at $45 \mathrm{mJy}^{\text {beam }}{ }^{-1}$ ), which is a common feature amongst many high-energy emitting pulsars (Abdo et al. 2010) and pulsar jets (Dodson et al. 2003; Bietenholz et al. 2004). The source PSR in IGR J11014-6103 shows standard properties of rotation powered pulsars and their associated PWNe (Possenti et al. 2002; Gotthelf 2004; Li et al. 2008), in terms of X-ray luminosity, power-law photon index, and stability over at least $30 \mathrm{yr}$ (Paper I), even if pulsations have not been detected so far (see Sect. 2.1.2; Paper I; T12). The similar luminosities inferred for the PSR and PWN are also in agreement with several observed PWN systems, and with the estimated system age of $1-2 \times 10^{4} \mathrm{yr}$ (Li et al. 2008). Furthermore, the pulsar spin-down power $\dot{E}_{\mathrm{PSR}}$ can be inferred from the total observed X-ray luminosity of the system (see, e.g., Li et al. 2008), $L_{\mathrm{X}} \approx 1.2 \times 10^{34} d_{7}^{2} \mathrm{erg} \mathrm{s}^{-1}$. This results in a rather high spin-down power $\dot{E}_{\text {PSR }} \sim 10^{37} \mathrm{erg} \mathrm{s}^{-1}$ for the PSR in IGR J11014-6103, in agreement with the observation of a bright PWN (Gaensler \& Slane 2006; Li et al. 2008). For a system with this spin-down power, the presence of the parent SNR should also be expected (Gaensler \& Slane 2006).

The PWN in the Lighthouse nebula displays a clear symmetry axis that defines the direction of motion of the system, pointing toward the center of the nearby SNR MSH 11-61A (see Fig. 1). This alignment, together with the similar distance derived from our $N_{\mathrm{H}}$ measure, further support the proposed association between the two objects (T12), and imply a pulsar speed ${ }^{1} v_{\text {PSR }} \sim(1100-2200) d_{7} \mathrm{~km} \mathrm{~s}^{-1}$. This kick velocity makes IGR J11014-6103 one of the most extreme runaway pulsars known so far. Further support for this high velocity comes from the observed geometry of the PWN. Following the analytical description of bow-shock wind confinements proposed by Wilkin (2000), a velocity of $\gtrsim 1000 \mathrm{~km} \mathrm{~s}^{-1}$ is required to match the PWN morphology, where the following parameters were adopted: $\dot{E}_{\text {PSR }} \sim 10^{37} \mathrm{erg} \mathrm{s}^{-1}$, an external medium particle density of $n_{\mathrm{ISM}}=0.1 \mathrm{~cm}^{-3}$, and an inclination $i=0^{\circ}$ of the velocity vector of the pulsar (not the inclination of the jet axis) with respect to the plane of the sky.

The observed shift between the X-ray and radio maxima $\left(\theta_{\text {peaks }} \sim 22^{\prime \prime}\right.$, see Fig. 3) can be used to estimate the PWN magnetic field. Taking a minimum PWN backflow velocity equal to the pulsar's speed $v_{\mathrm{PSR}} \approx 1000 \mathrm{~km} \mathrm{~s}^{-1}$, it takes a time $t_{\text {peaks }} \sim \theta_{\text {peaks }} d / v_{\text {PSR }}$ for particles to travel from the X-ray peak to the radio peak ( $d$ is the distance to IGR J11014-6103). If the same particle population is responsible for the emission at both energies, the synchrotron loss timescale is constrained to be $t_{\text {peaks }} \gtrsim t_{\text {sync }}\left(E_{\mathrm{e}^{-}}, B_{\mathrm{PWN}}\right) \approx 422 E_{\mathrm{e}^{-}}^{-1} B_{\mathrm{PWN}}^{-2} \mathrm{~s}$, where $E_{\mathrm{e}^{-}}$is the energy of the emitting electrons and $B_{\mathrm{PWN}}$ is the magnetic field in the nebula. Synchrotron emission at frequencies $h v_{\mathrm{c}}=5 \mathrm{keV}$ requires, on the other hand, that $h v_{5 \mathrm{keV}} \approx 5.2 B_{\mathrm{PWN}} E_{\mathrm{e}^{-}}^{2}$. Putting these constraints together, a minimum value $B_{\mathrm{PWN}} \approx 10-20 \mu \mathrm{G}$ is required, in good agreement with known PWN magnetic fields (Gaensler \& Slane 2006).

The PWN X-ray and radio spectra obtained in Sect. 2 imply the existence of a break frequency in between the two energy bands. Extrapolating the radio and X-ray power-law-like spectra, such a break should fall at $v_{\mathrm{br}} \sim 5 \times 10^{11} \mathrm{~Hz}$. This break frequency could be used, in principle, to further constrain the emitting electron distribution and/or the PWN magnetic field. However, the spectral break $\Delta \alpha \sim 0.92$, is much larger than the expected value of 0.5 in a single-population synchrotronemission scenario. A similar situation is observed in several PWN systems in which additional (still unclear) processes may need to be considered for an accurate derivation of the properties of the electron distribution and the nebular magnetic field (Gaensler \& Slane 2006; see also Reynolds 2009).

The distance $d \approx 7 \mathrm{kpc}$ derived for the Lighthouse nebula implies an intrinsic length of the main jet $l_{\text {jet }} \geq 11 \mathrm{pc}$, making it the longest X-ray jet detected so far in our Galaxy (accounting for projection further increases this estimate by a factor $(\sin \theta)^{-1}$, where $\theta \in[0, \pi / 2]$ is the jet angle to the line of sight, with a most likely value $\theta \sim 50^{\circ}$, see Sect. 2.3). The observed precession-like helical modulation, similar to that seen, e.g., in other galactic jets (Stirling et al. 2002; Durant et al. 2013), promptly suggests a ballistic jet origin for this structure. The different X-ray brightness and elongation of the jet and counterjet can also be easily reconciled in this scenario, owing to their different orientation with respect to the observer. To further characterize the ballistic pulsar jet scenario, we used a simplified helical model describing the large-scale structure of the jet. The model describes the data well, both in terms of the spatial modulation and the jet brightness profile (see Fig. 6), thus confirming the three-dimensional helical structure and consequently the

1 The velocity derived here for IGR J11014-6103 differs from the one reported in T12, as we adopted the refined distance of $7 \mathrm{kpc}$ for SNR MSH 11-61A reported in García et al. (2012). 
truly ballistic jet nature of the feature. An interpretation in a diffusion scenario of particles in the interstellar medium (Bandiera 2008) can be excluded, as it would require an underlying helical structure for the interstellar medium's magnetic field, which is instead known to be dominated by its turbulent component at scales below 100 pc (Gaensler et al. 2011; Giacinti et al. 2012).

The helical pattern seen along the jet could be explained either by free precession of the pulsar (with a period of $\sim 66 \mathrm{yr}$; see Sect. 2.3) or by the development of kink instabilities (see, e.g., Lyubarskii 1999). In this latter case, the period observed in the helical model would be associated with the timescale at which successive kinks appear. However, a low level of periodicity should be expected in this scenario, unless the instability is triggered by precession (Durant et al. 2013). Since our data reveal only approximately 1.5 helical steps along the main jet, such periodicity level in the trigger of the kink instabilities is difficult to assess. Periodic modulation has, on the other hand, been tentatively interpreted in some systems as free precession of the pulsar (see, e.g., Akgün et al. 2006; Jones 2012; Durant et al. 2013, and references therein), with periods up to several years (see, e.g. Weisberg et al. 2010). We note, however, that periods as long as several tens of years could hardly be detected in systems which do not show an extended jet, as in these systems the periodic modulation must be inferred by variations of the pulsar properties (e.g., spectral and timing properties, or of the linear polarization angle), based on monitoring of the source typically over timescales of a few years.

A free pulsar precession could be explained assuming a given value for the oblateness $(\epsilon)$ of the rotating neutron star (see e.g. Jones 2012), which can be estimated from the pulsar's spin and the precession period as $\epsilon=\left(I_{3}-I_{1}\right) / I_{1}=P_{\text {spin }} / P_{\text {prec }}$ (where $I_{1}$ and $I_{3}$ are principal moments of inertia; see, e.g., Haberl et al. 2006). For comparison with the values inferred for several likely precessing isolated pulsars, in the range $\epsilon \approx 10^{-4}$ to $10^{-10}$ (Jones \& Andersson 2001; Jones 2012; Durant et al. 2013), we compute here the oblateness of IGR J11014-6103. A rough estimate for the spin period of PSR is obtained considering the pulsar age (10-20 kyr) and spin-down energy estimated above, $\dot{E}_{\mathrm{PSR}} \sim 10^{37} \mathrm{erg} \mathrm{s}^{-1}$, which provides $P_{\text {spin }}$ of the order of $0.1 \mathrm{~s}$. A low value of the oblateness parameter is, therefore, obtained for the pulsar in the Lighthouse nebula, $\epsilon \sim 0.5 \times 10^{-10}$, which is close to values found in other cases (see above).

In our simulations, we did not consider any bending of the precessing axis, since it is not apparent in our Chandra images. The lack of bending implies that the curvature radius of the jet has to be large, $R_{\text {curv }} \gtrsim l_{\text {jet }}$. Since $R_{\text {curv }} \propto \dot{E}_{\text {jet }} R_{\text {jet }}^{-2} \rho_{\text {ISM }}^{-1} v_{\text {PSR }}^{-2}$, where $\dot{E}_{\text {jet }}$ is the jet power, $R_{\text {jet }}$ its radius, and $\rho_{\text {ISM }}$ is the medium mass density (see, e.g., Soker \& Bisker 2006, and references therein), it follows that the jet has to be rather powerful and/or collimated. A high jet power is indeed supported by the relative distribution of the total X-ray luminosity of the system. The jet contribution is similar to that of the pulsar and the PWN, suggesting that a sizable fraction of $\dot{E}_{\mathrm{PSR}}$ is channeled through the jets.

The jet X-ray spectrum suggests a synchrotron origin for its emission (see Sect. 2), with a relatively hard photon index $\Gamma=1.6$. Assuming that the radio and $\mathrm{X}$-ray emission are produced by a single electron population, a straight extension of the $\mathrm{X}$-ray spectrum would yield a flux $\sim 10^{-16} \mathrm{erg} \mathrm{cm}^{-2} \mathrm{~s}^{-1}$ at $2 \mathrm{GHz}$, for a bandwidth of $2 \mathrm{GHz}$. This estimate, being lower than the upper limit $\sim 4 \times 10^{-15} \mathrm{erg} \mathrm{cm}^{-2} \mathrm{~s}^{-1}$ obtained with ATCA at the same frequency, can naturally explain the radio nondetection of the jet.
The absence of breaks in the X-ray spectrum all along the jet implies that no significant cooling of the X-ray emitting electrons is taking place. We use the condition $h v_{\text {sync }} / 5 \mathrm{keV} \equiv$ $h v_{5 \mathrm{keV}} \approx 5.2 B_{\mathrm{jet}} E_{\mathrm{e}^{-}}^{2}$ again, require that the synchrotron timescale $t_{\text {sync }}$ is at least equal to the time taken by electrons to travel up to the jet tip, $\sim l_{\text {jet }} / v_{\text {jet }}$, and thereby obtain a maximum value for the jet magnetic field $B_{\text {jet }} \lesssim$ $65\left(h v_{5 \mathrm{keV}}\right)^{-1 / 3}\left(l_{\text {jet }} / 15 \mathrm{pc}\right)^{-2 / 3}\left(v_{\text {jet }} / 0.8 c\right)^{2 / 3} \mu \mathrm{G}$. A further constraint on $B_{\text {jet }}$ is derived from the requirement that electrons are confined within the jet so that their Larmor radius $r_{\mathrm{L}} \approx 1.75 \times$ $10^{25} E_{\mathrm{e}^{-}} B_{\text {jet }}^{-1} \mathrm{~cm}$ is at most equal to $R_{\text {jet }} \sim 2.3 \times 10^{17} d_{7} \mathrm{~cm}$. Putting these constraints together, the jet magnetic field is found to be $B_{\text {jet }} \sim 10-65 \mu \mathrm{G}$. Assuming instead near-equipartition between particles and magnetic fields (see, e.g., Pacholczyk 1970), we find $B_{\text {jet }}^{\text {equip }} \approx 15 \mu \mathrm{G}$, implying a minimum jet power $\dot{E}_{\text {jet }}^{\text {equip }} \approx$ $2 \times 10^{35} \mathrm{erg} \mathrm{s}^{-1}$. This estimate does not account, however, for the presence of relativistic protons, if any, and/or thermal material in the jet, which would make the total jet kinetic luminosity higher, in line with the high relative contribution (nearly 1/3) of the jets to the total system's luminosity and with the absence of any noticeable jet bending.

Although jet-launching mechanisms in pulsars are not yet fully understood, they likely have a magneto-hydrodynamical origin (Komissarov \& Lyubarsky 2004). The ultrarelativistic speed and the anisotropy of the pulsar wind (Bogovalov \& Khangoulian 2002) suggest that the jets are formed by magnetic hoop stresses onto the wind material downstream of the termination shock (Lyubarsky 2002). This termination shock, for the same conditions of the external medium, is located much closer to the neutron star in a high-velocity pulsar as compared to slow-moving systems (Gaensler \& Slane 2006). This could explain the relatively high jet power and collimation degree in the Lighthouse nebula, compared, e.g., to those from the Crab (Weisskopf et al. 2000) and Vela pulsars (Pavlov et al. 2003).

\section{Concluding remarks}

The Lighthouse nebula is the first case in which a jet from a hyperfast runaway pulsar can be clearly identified. The associated SNR MSH 11-61A is the remnant of a core-collapse explosion from a massive star (García et al. 2012). These types of supernovae are expected to produce in some cases neutron stars with natal kick velocities up to or exceeding $1000 \mathrm{~km} \mathrm{~s}^{-1}$ (Wang et al. 2007; Janka 2012). Simulations of core-collapse mechanisms however have difficulties in predicting orthogonal alignment between the pulsar spin-axis and the direction of motion for the highest-velocity systems, as observed in IGR J11014-6103 (Wang et al. 2007). Alternative models accounting for this misalignment have been suggested (see, e.g., Colpi \& Wasserman 2002; Davies et al. 2002), but these require an extreme rotation of the iron core of the presupernova star, not commonly accepted to be achievable (Fryer \& Warren 2004).

We remark that for the Guitar nebula (Hui et al. 2012), the pulsar's jet nature of the X-ray linear feature is not proven yet, and the originating SNR is not known so far. Nevertheless, if the feature will be confirmed as a true pulsar's jet, the supernova responsible for the system could already constitute the second example for such an unusual core-collapse episode, similar to the one originating the Lighthouse nebula.

Core-collapse supernovae are also expected to produce bipolar outflows during or right after the explosion (Davies et al. 2002; Paragi et al. 2010; Janka 2012). These events can, under certain circumstances, even lead to the formation of 
long-duration gamma-ray bursts (Davies et al. 2002; Paragi et al. 2010; Soderberg et al. 2010). The SNR MSH 11-61A shows a clear bipolar asymmetry along the NW-SE direction (García et al. 2012), which could be the imprint of the past outflow activity during the supernova explosion (Paragi et al. 2010; Janka 2012). The high kick velocity of IGR J11014-6103 and the rough alignment of its jets with the direction of the bipolar asymmetries in SNR MSH 11-61A could then be the echoes of a quenched long-duration gamma-ray burst.

Acknowledgements. This paper is based on Chandra and ATCA observations. We thanks prof. D. Lai for useful discussion, and the Chandra Uplink Support team, in particular Dr. J. Connelly, for the support in preparing the observation. L.P. thanks the Société Académique de Genève and the Swiss Society for Astrophysics and Astronomy for travel grants sustaining the ongoing collaboration between the ISDC and the ATCA team.

\section{References}

Abdo, A. A., Ackermann, M., Ajello, M., et al. 2010, ApJS, 187, 460 Akgün, T., Link, B., \& Wasserman, I. 2006, MNRAS, 365, 653

Arnaud, K., A. 1996, in ASP Conf. Ser. 101, eds. G. Jacoby, \& J. Barnes, 17 Bandiera, R. 2008, A\&A, 490, L3

Bietenholz, M. F., Hester, J. J., Frail, D. A., \& Bartel, N. 2004, ApJ, 615, 794

Bogovalov, S. V., \& Khangoulian, D. V. 2002, MNRAS, 336, L53

Bogovalov, S., \& Tsinganos, K. 1999, MNRAS, 305, 211

Carter, C., Karovska, M., Jerius, D., Glotfelty, K., \& Beikman, S. 2003, in Astronomical Data Analysis Software and Systems XII, eds. H. E. Payne, R. I. Jedrzejewski, \& R. N. Hook, ASP Conf. Ser., 295, 477

Colpi, M., \& Wasserman, I. 2002, ApJ, 581, 1271

Cordes, J. M., Romani, R. W., \& Lundgren, S. C. 1993, Nature, 362, 133

Davies, M. B., King, A., Rosswog, S., \& Wynn, G. 2002, ApJ, 579, L63

Davis, J. E., Bautz, M. W., Dewey, D., et al. 2012, in Proc. SPIE, 8443, 1A

De Luca, A., Marelli, M., Mignani, R., et al. 2011, ApJ, 733, 104

De Luca, A., Mignani, R. P., Marelli, M., et al. 2013, ApJ, 765, L19

Dodson, R., Lewis, D., McConnell, D., \& Deshpande, A. A. 2003, MNRAS, 343, 116

Durant, M., Kargaltsev, O., Pavlov, G. G., Kropotina, J., \& Levenfish, K. 2013, ApJ, 763, 72

Freeman, P., Doe, S., \& Siemiginowska, A. 2001, in Proc. SPIE 4477, eds. J.-L. Starck, \& F. D. Murtagh, 76

Fruscione, A., McDowell, J. C., Allen, G. E., et al. 2006, in Proc. SPIE, 6270, $1 \mathrm{~V}$

Fryer, C. L., \& Warren, M. S. 2004, ApJ, 601, 391
Gaensler, B., \& Slane, P. 2006, ARA\&A, 44, 17

Gaensler, B. M., Haverkorn, M., Burkhart, B., et al. 2011, Nature, 478, 214

García, F., Combi, J. A., Albacete-Colombo, J. F., et al. 2012, A\&A, 546, A91

Giacinti, G., Kachelrieß, M., \& Semikoz, D. V. 2012, Phys. Rev. Lett., 108, 1101

Gooch, R. 1996, in Astronomical Data Analysis Software and Systems V, eds.

G. H. Jacoby, \& J. Barnes, ASP Conf. Ser., 101, 80

Gotthelf, E. V. 2004, in Young Neutron Stars and Their Environments, eds.

F. Camilo, \& B. M. Gaensler, IAU Symp., 218, 225

Gotthelf, E. V., \& Halpern, J. P. 2008, ApJ, 681, 515

Haberl, F., Turolla, R., de Vries, C. P., et al. 2006, A\&A, 451, L17

Hui, C. Y., Huang, R. H. H., Trepl, L., et al. 2012, ApJ, 747, 74

Janka, H.-T. 2012, Ann. Rev. Nucl. Part. Sci., 62, 407

Johnson, S. P., \& Wang, Q. D. 2010, MNRAS, 408, 1216

Johnston, S., Kramer, M., Karastergiou, A., et al. 2007, MNRAS, 381, 1625

Jones, D. I. 2012, MNRAS, 420, 2325

Jones, D. I., \& Andersson, N. 2001, MNRAS, 324, 811

Kargaltsev, O., \& Pavlov, G. G. 2008, AIP Conf. Proc., 983, 171

Komissarov, S. S., \& Lyubarsky, Y. E. 2004, MNRAS, 349, 779

Lai, D., Chernoff, D. F., \& Cordes, J. M. 2001, ApJ, 549, 1111

Li, X.-H., Lu, F.-J., \& Li, Z. 2008, ApJ, 682, 1166

Lyubarskii, Y. E. 1999, MNRAS, 308, 1006

Lyubarsky, Y. E. 2002, MNRAS, 329, 34

Marelli, M., De Luca, A., Salvetti, D., et al. 2013, ApJ, 765, 36

Migliari, S., Fender, R., \& Méndez, M. 2002, Science, 297, 1673

Pacholczyk, A. G. 1970, Radio astrophysics. Nonthermal processes in galactic and extragalactic sources (San Francisco: Freeman)

Paragi, Z., Taylor, G. B., Kouveliotou, C., et al. 2010, Nature, 463, 516

Pavan, L., Bozzo, E., Pühlhofer, G., et al. 2011, A\&A, 533, A74 (Paper I)

Pavlov, G. G., Teter, M. A., Kargaltsev, O., \& Sanwal, D. 2003, ApJ, 591, 1157

Possenti, A., Cerutti, R., Colpi, M., \& Mereghetti, S. 2002, A\&A, 387, 993

Reynolds, S. P. 2009, ApJ, 703, 662

Reynoso, E. M., Johnston, S., Green, A. J., \& Koribalski, B. S. 2006, MNRAS, 369, 416

Roberts, M. S. E. 2004, The Pulsar Wind Nebula Catalog (March 2005 version), McGill University, Montreal, Quebec, Canada, available at http: //www.physics.mcgill.ca/ pulsar/pwncat.html

Sault, R., Teuben, P., \& Wright, M. 1995, ASP Conf. Ser., 77, 433

Soderberg, A. M., Chakraborti, S., Pignata, G., et al. 2010, Nature, 463, 513

Soker, N., \& Bisker, G. 2006, MNRAS, 369, 1115

Spruit, H., \& Phinney, E. S. 1998, Nature, 393, 139

Stirling, A. M., Jowett, F. H., Spencer, R. E., et al. 2002, MNRAS, 337, 657

Tomsick, J. A., Bodaghee, A., Rodriguez, J., et al. 2012, ApJ, 750, L39 (T12)

Wang, C., Lai, D., \& Han, J. L. 2007, ApJ, 656, 399

Weisberg, J. M., Everett, J. E., Cordess, J. M., Morgan, J. J., \& Brisbin, D. G. 2010, ApJ, 721, 1044

Weisskopf, M. C., Hester, J., Tennat, A. F., et al. 2000, ApJ, 536, L81

Wilkin, F. P. 2000, ApJ, 532, 400 